\title{
Reabilitação oral com Facetas de Resina Composta e influencia na qualidade de vida- Relato de Caso
}

Oral Rehabilitation with Composite Resin Facets and the influence on quality of life - Case Report Rehabilitación oral com Facetas de Resina Compuesta y la influencia em la calidad de vida - Relato de Caso

\section{Resumo}

A perda de um elemento dental, afeta significativamente a qualidade de vida de maneira funcional e estética, causando limitações sociais. Consequentemente a procura por uma melhora estética é possibilitada por várias formas de tratamento, sendo uma delas a resina composta que permite um resultado satisfatório, com praticidade e eficácia. Com isso, o presente trabalho consiste em um relato de caso clínico referente ao impacto da reabilitação oral na qualidade de vida por meio de facetas em resina composta, com auxílio de modelos deestudo através do enceramento diagnóstico e guia de silicone. O tratamento mostrou cumprir sua função satisfatoriamente ao recuperar a harmonia do sorriso, devolvendo ao paciente a estética objetivando a cumprir as expectativas e devolver a função mastigatória de maneira rápida e com um baixo custo.

Palavras-chave: Facetas dentárias; Reabilitação bucal; Resina composta.

\footnotetext{
Abstract

The loss of a dental element significantly affects the quality of life functionally and aesthetically, causing social limitations. Consequently, the search for an aesthetic improvement is made possible by various forms of treatment, one of them being the composite resin that allows a satisfactory result with practicality and effectiveness. This paper consists of a clinical case report on the impact of oral rehabilitation on quality of life through composite resin
} 
veneers, with the aid of study models through diagnostic wax-up and silicone guide. The treatment proved to fulfill its function adequately by restoring smile harmony, giving back to the patient the aesthetics aimed at fulfilling expectations and returning masticatory aim quickly and at a low cost.

Keywords: Dental veneers; Oral rehabilitation; Composite resin.

\section{Resumen}

The loss of a dental element significantly affects the quality of life in a functional and aesthetic manner, causing social limitations. Consequently the search for an aesthetic improvement is made possible by various forms of treatment, one of which is the composite resin that allows a satisfactory result, with practicality and effectiveness. El presente trabajo consiste en un informe de caso clínico sobre el impacto de la rehabilitación oral en la calidad de vida a través de facetas de resina compuesta, con la ayuda de modelos de estudio a través de la depilación diagnóstica y la guía de silicona. El tratamiento demostró cumplir su función satisfactoriamente recuperando la armonía de la sonrisa, devolviendo al paciente la estética con el objetivo de cumplir con las expectativas y devolver la función masticatoria de forma rápida y con un bajo coste.

Palabras clave: Carillas dentales; Rehabilitación oral; Compuesto.

\section{Introdução}

Atualmente tem se recorrido à odontologia não só pelo tratamento da dor, mas tambémpela procura de dentes brancos, bonitos e saudáveis de forma mais acessível e rápida. A perda de um elemento dental está associada a impactos estéticos, funcionais, psicológicos e sociais, como também um problema de saúde pública, já que atinge diretamente a autoestima do indivíduo (Kreve \& Anzolin, 2016).

A função mastigatória precária causa deglutição de pedaços muito grandes de alimentos ou em mudanças significativas da dieta, fazendo com que se evitem alimentos mais difíceis de serem mastigados e causando uma restrição alimentar (Paraguassu et al, 2019).

A estética ocupa um lugar de grande destaque na Odontologia moderna, enfocando procedimentos cada vez mais próximos da perfeição e capazes de imitar a natureza, mantendotamanho, forma, cor e simetria. Atentos a essa nova filosofia, os pacientes almejam cada vez mais, um sorriso esteticamente agradável com dentes claros e alinhados (Santos et al, 2016). Alterações na estética facial acarretadas por perda dentária interferem na autoestima e nos relacionamentos interpessoais, podendo deixar o indivíduo intimidado e retraído (Nepomuceno et al, 2019).

Reabilitar é recuperar ou melhorar a saúde bucal do paciente, quando esta se encontracomprometida (Silva et al, 2017). As opções de tratamentos reabilitadores vão desde as próteses parciais removíveis, totais, fixas, próteses sobre implantes, até as facetas ou laminados em cerâmicaou resinas compostas (Dallazen et al, 2015).

A técnica de reabilitação utilizando a resina composta destaca-se pela rapidez, segurança e eficácia. Além disso, dispensa etapas laboratoriais e não requer confecção de provisório As resinas compostas de uso direto são materiais indicados para casos que necessitem de alterações na anatomia dental, tais como correções de ângulos incisais, bossas e sulcos de desenvolvimento fora dos padrões, ou ainda alterações morfológicas de dentes conóides ou diastemas (Gouveia et al, 2018).

As facetas representam uma alternativa restauradora estética que têm como possibilidade minimizar o desgaste dentário necessário durante a fase do preparo, e proporcionar uma excelente harmonia do sorriso (Moreira et al, 2019). As restaurações diretas com resina composta resultam em procedimentos minimamente invasivos e de máxima preservação da estrutura dental (Gouveia et al, 2018; Oliveira et al, 2021).

Adicionalmente, Lima et al, (2013) observaram que o uso de resinas compostas é uma alternativa conservadora que restitui a saúde biológica e psicológica de pacientes de forma rápida. Esse resultado está alinhado à atual preocupação com o restabelecimento da estética dental, uma vez que um sorriso harmônico é almejado pela maioria dos pacientes que procuram o consultório odontológico (Moreira et al, 2018; Campos et al, 2021). 
Nos últimos anos, a estética tem obtido bastante êxito na procura pelos pacientes, pois além de proporcionar uma saúde bucal adequada, promove satisfação ao paciente diante do seu sorriso. Esse senso de estética está relacionado diretamente à promoção do aumento da autoestima, pois o sorriso tornou-se um grande acessório para agradar os recursos visuais; integrando a reabilitação, que se torna essencial por restabelecer ou executar reparos na forma, posicionamento e o equilíbrio estético e funcional dos dentes (Santos et al, 2016).

Diante do exposto, este trabalho objetivou relatar por meio de um caso clínico a influência de uma reabilitação oral estética com resinas compostas na qualidade de vida do indivíduo.

\section{Metodologia}

$\mathrm{O}$ estudo tratou de um relato de caso de um paciente atendido na Faculdade de Odontologia do Recife. Foi realizado o exame clínico e físico do paciente. Foi proposto para o paciente o tratamento com facetas em resina para a reabilitação oral da mesma. Todos os aspectos éticos foram respeitados na elaboração deste caso clinico. O paciente foi instruído sobre a necessidade de enriquecer a comunidade acadêmica com novos estudos sobre o caso em questão. O paciente assinou um Termo de Consentimento Livre e Esclarecido para permitir sua participação no estudo. Os responsáveis pelo desenvolvimento do caso respeitaram a Declaração de Helsinque para cumprimento dos aspectos éticos. Além disso, o trabalho foi submetido ao Comitê de Ética em Pesquisa da Universidade de Pernambuco (CEP) e aprovado pelo parecer de número 4.748.525.

Para a sustentação do desenvolvimento do caso de forma correta, foram consultados artigos, livros e periódicos nas bases de dados PUBMED/MEDLINE; LILACS e SCIELO. Os descritores utilizados para a pesquisa foram "facetas em resina"; "reabilitação oral" e "resina composta". Foram incluídos relatos de caso independente da data de publicação, além disso também foram incluídos revisões sistemáticas e meta-análises, e estudos que abordavam especificamente a técnica facetas em resina composta.

\section{Relato do Caso}

Paciente do sexo masculino, compareceu à clínica de multiespecialidade da Faculdade de Odontologia do Recife insatisfeito com a harmonia do seu sorriso, o mesmo foi encaminhado pela clínica do curso de implante na FOR, onde já tinha iniciado e estava em tratamento para confecção de um implante no elemento 11.

Durante a anamnese e exame físico observou-se que o paciente havia restabelecidohá pouco tempo as guias caninas e apresentava desgaste nos incisivos superiores e inferiores. Logo após foi elaborado um plano de tratamento que se adequasse às necessidades e condições do paciente. Na sequência foi mostrado e explicado ao paciente o plano de tratamento proposto para sua concordância e o termo de consentimento livre esclarecido para que pudesse assiná-lo.

Após a fase de adequação do meio bucal, por meio de orientações de higiene, foi realizado, primeiramente, o registro fotográfico (Figura 1,2 e 3). Logo após foi realizada a moldagem dos arcos superiores e inferiores e confecção de modelos de gesso. Foram feitos os registros do paciente com o arco facial, a confecção do jig com godiva junto ao registro intermaxilar com a cera 7 para montagem em articulador semi ajustável (ASA). Depois foi realizado o enceramento diagnóstico para estudo e planejamento do caso (Figura 4 e 5). 
Figuras 1, 2 e 3: Registro inicial.
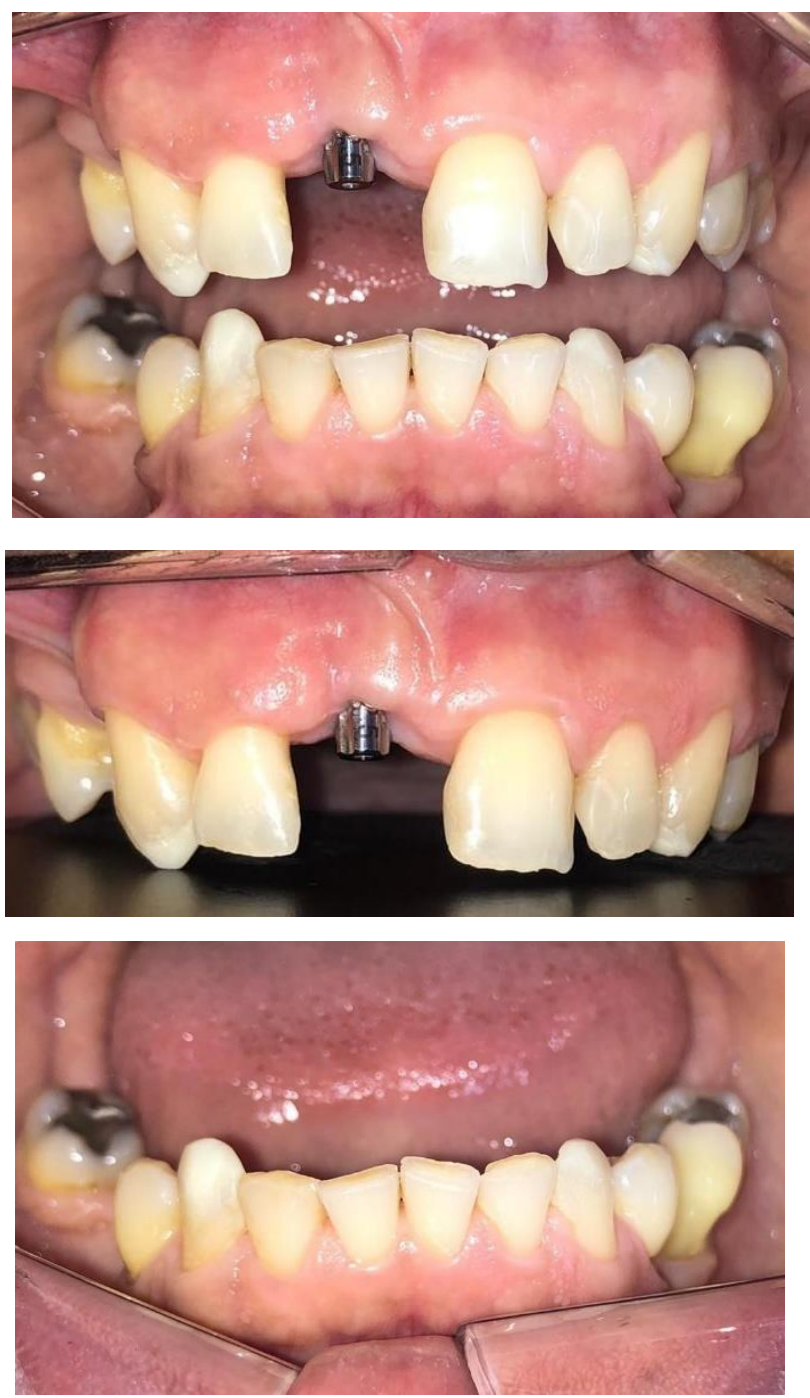

Fonte: Autores.

Foi iniciado o tratamento com a confecção das facetas nos elementos inferiores, foi feita uma guia palatina de silicone de condensação (Precise SX - Dentsply) para auxiliar no processo restaurador, trazendo mais previsibilidade e segurança, foi feita a escolha da resina eda cor correspondente (Charisma diamond - Kulzer) na cor (A2). Foi escolhido o isolamento absoluto modificado neste caso para poder ajustar a guia de silicone, logo após foi realizado ocondicionamento ácido dos elementos com ácido fosfórico a 37\% ( Condac 37 - FGM) por 15segundos, lavada a superfície dental com água em abundância e seca com jatos de ar, depois foi feita a aplicação de duas camadas do sistema adesivo (Ambar APS - FGM ) de forma ativa esfregando com auxílio de um microaplicador ( Cavibrush - FGM) por 10 segundos cada camada e fotoativado por 20 segundos usando o aparelho fotopolimerizador da Gnatus, confeccionando as facetas nos elementos 31,32,41,42 com a resina de forma incremental realizando a escultura com espátulas de resina e pincel decidindo realizar o polimento na próxima consulta (Figura 6). 
Figuras 4 e 5: Enceramento diagnóstico
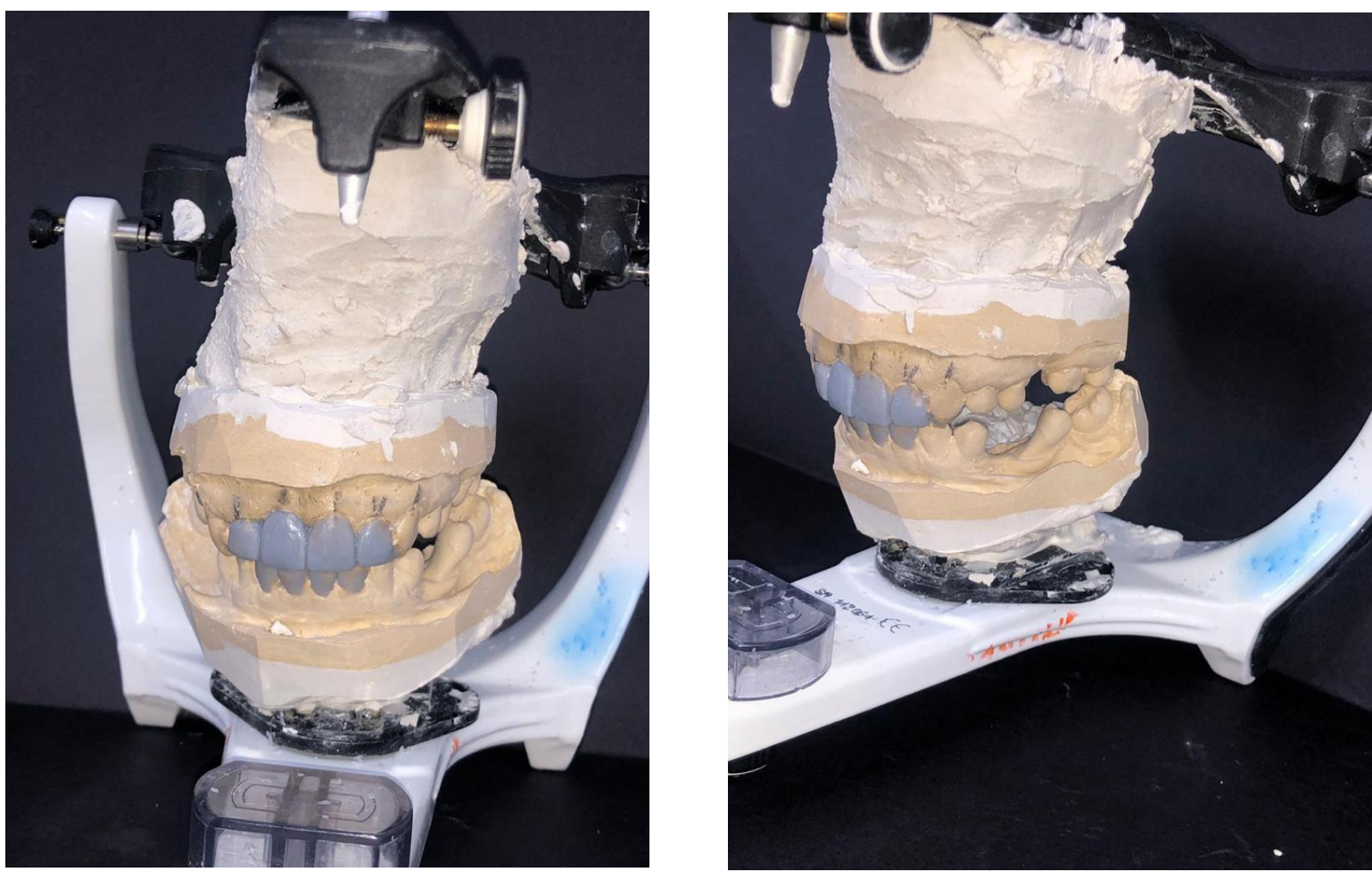

Fonte: Autores.

Figura 6: Após confecção das facetas nos elementos inferiores.

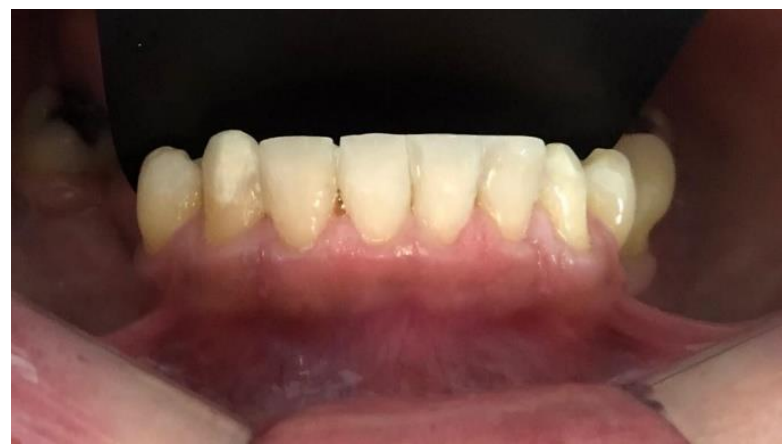

Fonte: Autores

Na consulta seguinte foi realizada a confecção das facetas nos elementos 12, 21 e 22 com o mesmo protocolo usado para os elementos inferiores. Em seguida foi realizado o ajusteoclusão com papel carbono e pinça muller, conferindo a correta oclusão, feito o acabamento para remoção de excessos com pontas diamantadas e lâmina de bisturi $\mathrm{n}^{\circ} 12$ seguido do polimento das restaurações com auxílio de discos de lixa (TDV), tira de lixa (Sof-Lex 3M), disco de feltro e pasta de polimento (TVD). Após a finalização foi realizado um novo registro fotográfico para a documentação. (Figura 7 e 8 )

Após o paciente finalizar o tratamento de implante que estava realizando em outro curso, foi chamado a outra consulta para realização da documentação fotográfica, obtendo assim o resultado final. (Figura 9 e 10). 
Figuras 7 e 8: Finalização das facetas.
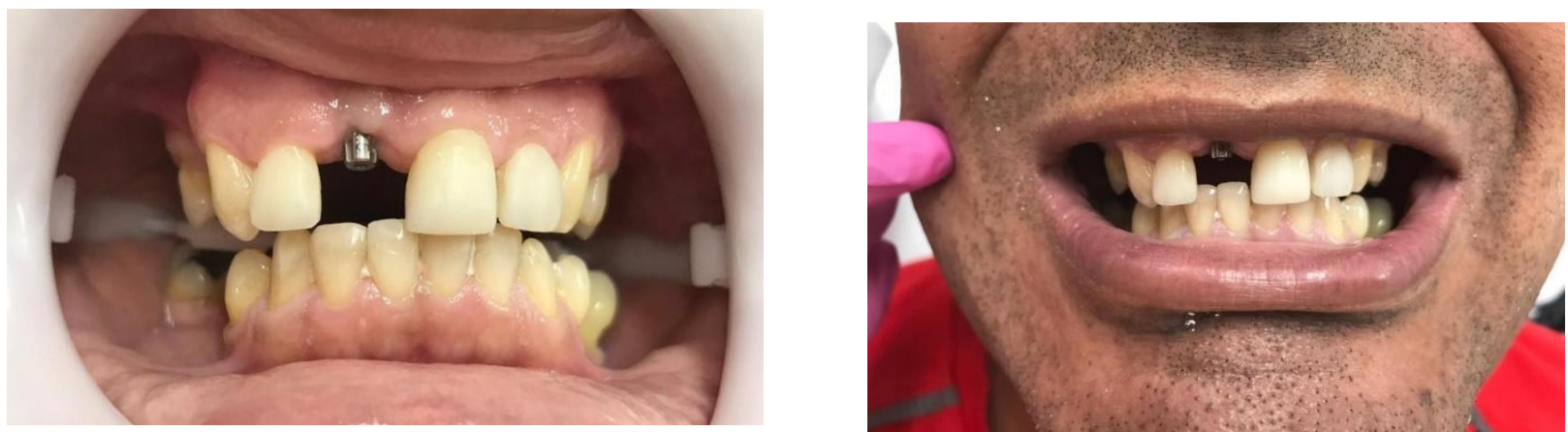

Fonte: Autores.

Figuras 9 e 10: Resultado final.
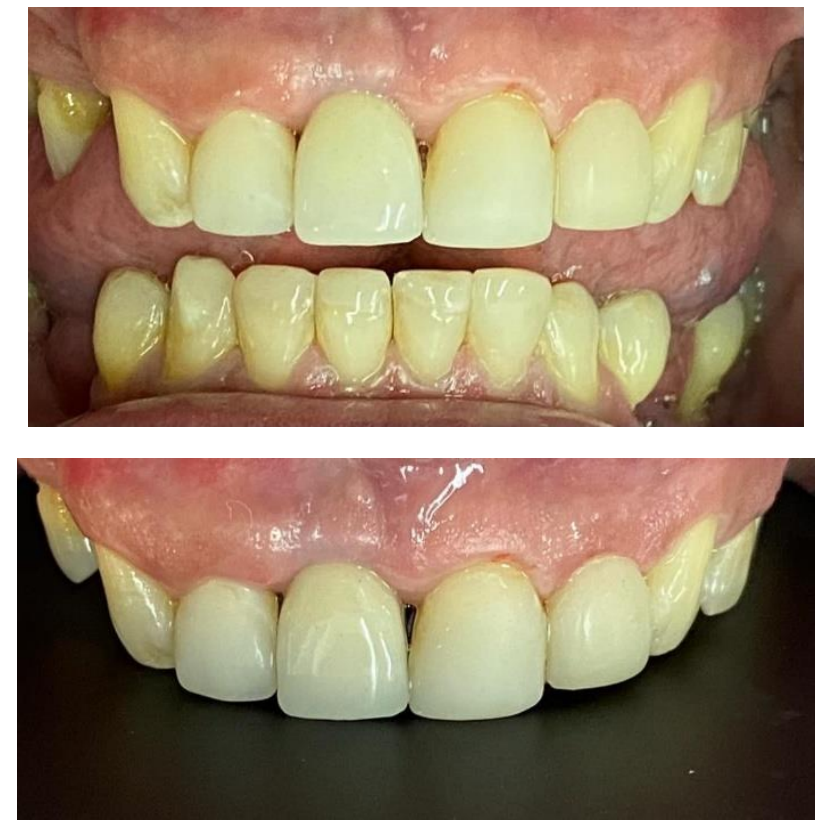

Fonte: Autores.

\section{Discussão}

A odontologia vem seguindo caminhos que vão além de apenas técnicas restauradoras, mas estabelece função, estética e bem estar do paciente de maneira geral, conseguindo assim devolver auto estima,prazer em sorrir e em viver (Mandarino, 2003). Nascimento et al (2018), compreendem em seu estudo que o tratamento reabilitador interfere não só na função mastigatória, mas também na estética da face e no sorriso, devolvendo a satisfação com o estado da boca, sendo este um fator diretamente ligadocom o psicológico do indivíduo, semelhante ao que Veyrune JL et al (2005) apresentam em seu estudo, onde a reabilitação melhora na qualidade de vida aumentando a autoestima, permitindo seu desenvolvimento social e reconstruindo sua imagem pessoal e social.

De acordo com Kreve e Anzolin (2016), a estética deve ser analisada sob dois pontos de vista: o da sociedade e o do próprio indivíduo, a visão individual do que é belo e estético. Sendo o sorriso bastante significativo, levando a conotações de alegria, bem-estar, satisfação, entre outras. Salientando a importância dos dentes nas relações empregatícias, sociais, culturais e na autoestima de uma pessoa. 
Por consequência, quando se realiza um tratamento reabilitador existe uma expectativa do paciente por um resultado estético nos padrões socialmente aceitáveis. Polsani,et al (2011), argumenta que para que esse desejo seja cumprido, o dentista deve estar ciente dos anseios e preocupações do paciente com sua aparência e se esforçar para atender as exigências estéticas.

Segundo Bastone et al (2000), o conceito de estética é subjetivo, sendo relacionado normalmente a beleza e harmonia, dependendo também de fatores sociais, culturais e psicológicos que devem ser levados em conta ao avaliar a satisfação do paciente com o resultado estético do tratamento. Assim, Flores, et al (2007) e Ferracane (2011) relatam que é imprescindível um planejamento criterioso e uma análise detalhada de cada passo do tratamento, pois seguindo esses critérios aumentam as chances de êxito estéticoe funcional do tratamento.

O caso em questão seguiu todos os passos planejados desde um exame clínico rigoroso ao planejamento por meio do enceramento diagnóstico, que permitiu uma previsãona execução da escultura dental tanto na oclusão quanto estética, levando a um resultado satisfatório. Assim como Dallazen et al (2015) relatam, o respeito e a execução de um criterioso planejamento inicial são os principais elementos para o êxito do tratamento. Os mesmos também comentam a existência de diferentes técnicas e a variedade de materiais que podem ser usados na reabilitação, permitindo ao profissional escolher a forma de conduzir o tratamento de acordo com cada caso.

O material escolhido para realizar esse caso foram as resinas compostas utilizadas na técnica direta, que como exposto por Gouveia, et al. (2017) é um material com propriedades físicas e mecânicas semelhante à estrutura dental, possuindo alta resistência mecânica à fratura e desgaste, excelente polimento superficial e boa estabilidade de cor. Semelhante a Hoeppner, et al (2003), que menciona mais algumas vantagens da técnica com resina composta como, podem ser feitas em apenas uma sessão, tem um preparo mais conservador, dispensa etapas laboratoriais, o custo é reduzido, tem um bom resultado estético,sendo seguro e eficaz. Sendo esses alguns dos motivos que baseiam a escolha deste material no estudo.

No entanto, o Mante et al (2013) também relata que podem ocorrer algumas falhas no uso da mesma, sendo as mais comuns formação de cárie secundária, desgaste, alteração de cor e sensibilidade pós operatória. Tais danos podem ser evitados seguindo a ordem correta dos passos e também utilizando a técnica correta do sistema adesivo.

Cardoso, et al (2011) e Hoeppner et al (2003) nomeiam alguns casos emque a resina composta deve ou não ser usada, sendo indicada para dentes conóides, fechamento de diastema, dentes escurecidos com necessidade de alteração de coloração,perdas estruturais por cárie, fraturas entre muitos outros. As resinas compostas mostram ser um material que além de qualidade, tem bastante versatilidade. Porém, não deve ser utilizada quando há uma perda estrutural muito extensa, quando o paciente tem uma oclusão tipo topo ou possui hábitos parafuncionais.

Junto a um consenso, a técnica escolhida para este caso foi a direta, onde dispensa a parte laboratorial, pois diminuiria os custos finais do tratamento. Segundo o que relata Hirata, et al (1999) a escolha é particular a cada situação, oferecendo ao profissional liberdade de escolher qual conduta seguir. O mesmo também relata algumas vantagens das técnicas diretas,em casos devidamente indicados, sendo a facilidade de manipulação do material, mais rapidezno tratamento, além de uma boa resistência mesmo que um pouco inferior à técnica indireta.

Seguindo a mesma linha de pensamento, Bispo (2009) menciona a desvantagem da técnica direta em relação a indireta, na estabilidade de cor e durabilidade nos aspectos estéticos, porém defende a técnica direta, pela praticidade, qualidade e custo benefício. Sendo assim, as duas técnicas são efetivas e com bons resultados, devendo ser indicadas corretamente a cada caso.

Outra escolha importante foi o tipo de resina utilizada, optando por uma nano-híbrida que, segundo o Minto (2018) é um material com excelentes propriedades físicas, mecânicas e estéticas, destacando-se na alta resistência mecânica , sendo um fator importante na escolha para a condução do caso apresentado. Como exposto também no estudo de Machado, et al 
(2016) as resinas nanohíbridas, promovem uma maior lisura superficial, melhorando na qualidade do polimento, sendo indicadas tanto em restaurações estéticasquanto em regiões de grande esforços mastigatórios.

O último passo do tratamento, após a finalização das esculturas, é o acabamento e polimento das restaurações. Machado, et al, (2016) comentam que é uma etapa fundamental para o sucesso e longevidade do tratamento. Menezes, et al (2014) relatam também que, quando bem realizados, o acabamento e polimento podem prevenir a rugosidade superficial, ausência de brilho e estabilidade na cor. O acabamento tem o objetivo de remover excessos de material e irregularidades promovendo assim melhor contorno anatômico e regularidade à superfície. Já no polimento tem-se a obtenção de brilho e lisura, removendo ranhuras geradas no acabamento, auxilia na redução do acúmulo de placa, manchas na superfície e na longevidade da restauração.

O caso clínico apresentado corrobora com os autores relatados, demonstrando a importância de um bom diagnóstico e planejamento para realização do tratamento com materiais e técnicas adequadas à resolução do problema proposto, como também de seguir corretamente os passos indicados para obter um resultado estético satisfatório, levando em conta as expectativas do paciente.

\section{Conclusão}

O tratamento reabilitador estético por meio de resinas compostas obtém resultados bastante satisfatórios, tendo também suas limitações, logo devem ser usadas após uma avaliação particular de cada caso, sendo importante um bom planejamento e diagnóstico por meio do enceramento diagnóstico. Assim, é uma opção de tratamento bastante viável considerando seu preparo mais conservador, sua longevidade, praticidade, baixo custo e o sucesso estético e funcional que ela promove.

\section{Referências}

Bastone, E. B., Freer, T. J., \& Mcnamara, J. R. (2000). Epidemiology of dental trauma: a review of the literature. Aust Dent J. 45(1): 2-9.

Bispo L. (2009) Facetas estéticas: Status da Arte. Rev Dentística online; 8(18): 11-14.

Cardoso P. C., Decursio R. A., Pacheco A. F. R., Monteiro L. J. E., Ferreira M. G., Lima P. L. A., \& Silva R. F. (2011). Facetas Diretas de Resina Composta e Clareamento Dental: Estratégias para Dentes Escurecidos. Rev. Odontol Bras Central. 20(55): 341-347

Campos, K. M. G. de., Rodrigues, R. A., Figueiredo, C. H. M. da C., Guenes, G. M. T., Alves, M. A. S. G., Rosendo, R. A., Penha, E. S. da., Nogueira, P. L., Dantas, M. V. O., \& Medeiros, L. A. D. M. (2021). Direct veneers anteriors: A literature review. Research, Society and Development, 10(6), e48910615729. https://doi.org/10.33448/rsd-v10i6.15729

Dallazen E., Bueno A. L. N., Araujo F. O., Milani P. A. P., \& Pupo Y. M. (2015). Alternativas de tratamento para reabilitação bucal estética e funcional. Rev Dental Press Estét. 12(2):51-6

Ferracane J. L (2011). Resin composite: state of the art. Dent Mater. 27(2): 29-38.

Flores M. T, Andersson L., Andreasen J. O., Baklnad L. K., Malmgren B., \& Barnett F. et al. (2007) Guidelines for the evaluation and mangement of traumatic dental injuries I. Fracturesand luxations of permanent teeth. Dent Traumatol. 23:66-71.

Gouveia C. G., Júnior R. M., Peralta F. S., Scherma A. P., \& Resende L. F. M (2018). Facetas Diretas de resina composta em dentes anteriores: relato de caso. ClipeOdonto. 9(1):44-50.

Gouveia T. H. N., Theobaldo J. D., Vieira-Junior W. F, Lima D., \& Aguiar F. H. B. (2017). Esthetic smile rehabilitation of anterior teeth by treatment with biomimetic restorative materials: acasereport. Clin Cosmet Investig Dent; 9:27-

Hirata R., \& Carniel C. Z. (1999). Solucionando alguns problemas clínicos comuns com o uso de facetamento direto e indireto: uma visão ampla. J Bras clin estet odontol, 3(15), 7-17. 1999 
Research, Society and Development, v. 11, n. 3, e23611326467, 2022

(CC BY 4.0) | ISSN 2525-3409 | DOI: http://dx.doi.org/10.33448/rsd-v11i3.26467

Hoeppner M. G., Pereira S. K., Siebel Neto E., \& De Camargo L. N. G. (2003). Tratamento estético de dente com alteração cromática: faceta estética em resina composta. Publ UEPG Biol Health Sc. 9(3/4): 67-72.

Kreve, S., \& Anzolin, D. (2016). Impacto da saúde bucal na qualidade de vidado idoso. Revista Kairós Gerontologia, 19, 45-59.

Machado A. C., Reinke A. C. M. A., Moura G. F., Zeola L. F., Costa M. M., Reis B. R., \& Soares P. V. (2016). Reabilitação estética e funcional com facetas diretas após histórico detraumatismo dento-alveolar. Rev Odontol Bras Central 25(74)

Mandarino F (2003). Cosmética em restaurações estéticas. http://www.forp.usp.br/restauradora/dentistica/temas/este_cosm/este_cosm.html,2003

Mante F. K., Ozer F., Walter R., et al (2013). The current state of adhesive dentistry: a guide for clinical practice. Compend Contin Educ Dent 34(9):2-8.

Menezes M. S., Vilela A. L. R., Silva F. P., Reis G. R., \& Borges M. G. (2014). Acabamento e polimento em resina composta: reprodução do natural Rev Odontol Bras Central. 23(66).

Moreira E. J. R, Neto J. A. F., \& Freitas G. C. (2018). Harmonização Estética do Sorriso com FacetasDiretas em Resina Composta: Relato de Caso. Sci Invest Dent 2018; 23(1):22-27

Moreira H. B., Assis H. P., Moreira J. P. V., \& Rodrigues C. (2019). Reabilitação Estética com Faceta Direta em Resina Composta.Revista Multidisciplinar Pey Këyo. 5(3)

Minto A. M. P. (2018). Reabilitação estético-funcional com resina composta Charisma® Diamond em paciente com erosão dental.

Nascimento J. E, Sales M. S. M., Ferreira E. F, Farias P. K. S., Ferreira R. C., \& Martins A. M. E. de B. (2018). Reabilitação com prótese dentária total em idosos e melhoria na dimensão do OHIP. Arq Odontol, 54.

Nepomuceno N. V. A., Machado C. T. A., Lima A. L. O., Ribeiro C. M. B., \& Vanderlei A. D (2019). Reabilitação Protética: sua influência na qualidade de vida. Revista da acBO. 28(1), 9-15

Oliveira, L. M. de O. e., Bezerra, R. N., Carmo, F. T. do., Meira, G. de F., Sá, J. L. de, \& Ramalho, L. O. (2021). Fechamento de diastema pela técnica direta em resina composta: relato de caso clínico. Research, Society and Development, 10(16), e431101624060. https://doi.org/10.33448/rsd-v10i16.24060

Paraguassu E. C., Figueira K. S., Lacerda J. P., Neto U. G. G., \& Gomes C. E. V (2019). Qualidade de vida e satisfação em usuários de prótese total no estado do Amapá, Brasil. REAS/EJCH. 27, e876.

Polsani L. R., Ajay Kumar G., Githanjali M., \& Anjana R. (2011). Geriatric Psychology and Prosthodontic Patient. Int J Prost Restorative Dentistry, $1(1), 1-5$.

Santos B. C., Dantas L. F., Silva S. C., Lima L. H. A., Agra D. M., \& Fernandes D. C. (2016). Odontologia Estética e Qualidade de Vida: Revisão Integrativa. Ciências Biológicas e da Saúde 3(3), 91-100.

Silva B. C. M., Ribeiro H. H. S., Viana H. C., Mendonça M. B., Silva G. R., \& Dietrich L. A. (2017) Importância da Reabilitação Oral Através da Prótese Parcial Removível: Relato de Caso. Revista de Odontologia Contemporânea. 1(2).

Veyrune J. L., Tubert-Jeannin S., Dutheil C., \& Riordan P. J (2005). Impact of new prostheses on theoral health related quality of life of edentulous patients Gerodontology. 22(1):3-9. 